\title{
Coupling of definitizable operators in Kreĭn spaces
}

\author{
V. Derkach ${ }^{1,2}$, C. Trunk ${ }^{3}$ \\ ${ }^{1}$ Department of Mathematics, Dragomanov National Pedagogical University, Pirogova 9, Kiev, 01601, Ukraine \\ ${ }^{2}$ Department of Mathematics, Vasyl Stus Donetsk National University, 600-Richchya Str 21, Vinnytsya, 21021, \\ Ukraine \\ ${ }^{3}$ Institut für Mathematik, Technische Universität Ilmenau, Postfach 100565, D-98684 Ilmenau, Germany \\ derkach.v@gmail.com, carsten.trunk@tu-ilmenau.de
}

DOI 10.17586/2220-8054-2017-8-2-166-179

\begin{abstract}
Indefinite Sturm-Liouville operators defined on $\mathbb{R}$ are often considered as a coupling of two semibounded symmetric operators defined on $\mathbb{R}^{+}$ and $\mathbb{R}^{-}$, respectively. In many situations, those two semibounded symmetric operators have in a special sense good properties like a Hilbert space self-adjoint extension.

In this paper, we present an abstract approach to the coupling of two (definitizable) self-adjoint operators. We obtain a characterization for the definitizability and the regularity of the critical points. Finally we study a typical class of indefinite Sturm-Liouville problems on $\mathbb{R}$.
\end{abstract}

Keywords: self-adjoint extension, symmetric operator, Krěn space, locally definitizable operator, coupling of operators, boundary triple, Weyl function, regular critical point.

Received: 18 January 2017

Revised: 1 February 2017

\section{Introduction}

Let $\mathcal{K}$ be a Hilbert space with the inner product $(\cdot, \cdot)$ and let $J$ be a linear operator in $\mathcal{K}$, such that $J=$ $J^{*}=J^{-1}$. The space $\mathcal{K}$ endowed with Hermitian sesquilinear form $[\cdot, \cdot]_{\mathcal{K}}=(J \cdot, \cdot)$ is called a Krĕn space and is denoted by $\left(\mathcal{K},[\cdot, \cdot]_{\mathcal{K}}\right)$, for details see $[1,2]$ or Section 2.1 below.

The Hermitian sesquilinear form $[\cdot, \cdot]_{\mathcal{K}}$ induces in an obvious way a sign type spectrum for linear operators. In the last two decades, this notion was frequently used in theoretical physics in connection with $\mathcal{P} T$-symmetric problems; here, we mention only [3-7] and in the study of $\mathcal{P} T$-symmetric operators, we refer to [8-11].

A self-adjoint operator $A$ in a Kreĭn space $\left(\mathcal{K},[\cdot, \cdot]_{\mathcal{K}}\right)$ is said to be definitizable [12], if its resolvent set $\rho(A)$ is nonempty and there exists a real polynomial $p$ such that $p(A)$ is nonnegative in $\left(\mathcal{K},[\cdot, \cdot]_{\mathcal{K}}\right)$. If $\alpha_{1}<\alpha_{2}<\cdots<\alpha_{N}$ is the set of all real zeros of $p$, then there exists a spectral function $E(\Delta)$ of $A$, which is defined on all intervals $\Delta$, such that the endpoints of $\Delta$ do not belong to the set $\left\{\alpha_{j}\right\}_{j=1}^{N}, E(\Delta)$ takes values in the set of orthogonal projections, commuting with $A$ and $E(\Delta)$ is monotone on each interval $\left(\alpha_{j}, \alpha_{j+1}\right)$. These intervals are classified in [12] as intervals of positive and negative type and the points $\alpha_{j}$ which are spectral points of neither positive type nor negative type are called critical, see exact denitions in Section 2.2. A critical point $\alpha$ is called regular, if the operators $E(\Delta)$ are uniformly bounded for all small $\Delta$ containing $\alpha$, otherwise it is called singular. The set of critical points of $A$ is denoted by $c(A)$, the set of regular (singular) critical points of $A$ is denoted by $c_{r}(A)$ $\left(c_{s}(A)\right.$, respectively). The notion of local definitizability of a self-adjoint operator $A$ in a Kreĭn space $\left(\mathcal{K},[\cdot, \cdot]_{\mathcal{K}}\right)$ was introduced in $[13,14]$, see Section 3 below.

In the present paper, the following problem is studied: the problem of the definitizability of the coupling $A$ of two symmetric operators $A_{+}$and $A_{-}$and the regularity of their critical points. Note the definition of the coupling from [15] adapted to the case of Krein spaces. Let a Kreĭn space $\left(\mathcal{K},[\cdot, \cdot]_{\mathcal{K}}\right)$ be the orthogonal sum $\mathcal{K}=\mathcal{K}_{+}[\dot{+}] \mathcal{K}_{-}$ of $(\mathcal{K},[\cdot, \cdot])$ of two Kreĭn spaces $\left(\mathcal{K}_{+},[\cdot, \cdot]_{\mathcal{K}_{+}}\right)$and $\left(\mathcal{K}_{-},[\cdot, \cdot]_{\mathcal{K}_{-}}\right)$, such that the subspaces:

$$
\mathcal{D}_{+}=\left\{f \in \mathcal{K}_{+} \cap(\operatorname{dom} A): A f \in \mathcal{K}_{+}\right\} \text {and } \mathcal{D}_{-}=\left\{f \in \mathcal{K}_{-} \cap(\operatorname{dom} A): A f \in \mathcal{K}_{-}\right\}
$$

are dense in $\mathcal{K}_{+}$and $\mathcal{K}_{-}$and the restrictions:

$$
A_{+}=\left.A\right|_{\mathcal{D}_{+}} \quad \text { and } \quad A_{-}=\left.A\right|_{\mathcal{D}_{-}}
$$

are symmetric operators with defect numbers $(1,1)$ in the Kreĭn spaces $\left(\mathcal{K}_{+},[\cdot, \cdot]_{\mathcal{K}}\right)$ and $\left(\mathcal{K}_{-},[\cdot, \cdot]_{\mathcal{K}}\right)$, respectively. The operator $A$ is called a coupling of two symmetric operators $A_{+}$and $A_{-}$. The coupling $A$ of two symmetric operators $A_{+}$and $A_{-}$is not uniquely defined by the above definition. We will make this definition more precise in Theorem 4.4 by using the boundary triple approach developed in [16-19]. For differential operators with indefinite weights, the coupling method was used in [20], and also in [21-23] to study the similarity problem and in [24] to study definitizabilty. 
The main result of the paper is Theorem 4.6, where conditions for regularity of the critical point $\infty \in c(A)$ are found under the assumptions that the symmetric operators $A_{+}$and $A_{-}$admit definitizable and semibounded extensions $A_{+, 0}$ and $A_{-, 0}$. The proof is based on the K. Veselic criterion of regularity [25,26] adapted to the case of definitizable operators in [27]. In the case when $A_{+}$and $A_{-}$are Hilbert space symmetric operators, similar results were obtained in [23] and [28].

Typically, such problems arise in the study of indefinite Sturm-Liouville operators:

$$
\ell(f)(t):=\frac{\operatorname{sgn} t}{w(t)}\left(-\frac{d}{d t}\left(\frac{d f}{r(t) d t}\right)+q(t) f(t)\right) \quad \text { for a.a. } \quad t \in \mathbb{R},
$$

where the coefficients $r, q$ and $w$ are real functions on $\mathbb{R}$ satisfying the conditions:

(C1) $r, q, w \in L_{\text {loc }}^{1}(\mathbb{R})$ and $r, w>0$ a.e. on $\mathbb{R}$,

(C2) the expression $\ell$ is in the limit point case at $-\infty$ and at $+\infty$,

(C3) minimal differential operators $B_{ \pm}$generated by $\pm \ell$ in $L_{w}^{2}\left(\mathbb{R}_{ \pm}\right)$are semibounded from below.

The operator $A$ generated by the differential expression (1.1) in the Krein space is the coupling of two semibounded symmetric operators $A_{ \pm}:= \pm B_{ \pm}$. In Proposition 5.1, it is shown that the operator $A$ is definitizable over a vicinity of $\infty$ and conditions (4.18) for $\infty \notin c_{s}(A)$ are formulated in terms of the $m$-coefficients for the operators $B_{ \pm}$. In the case $w \equiv 1$, the conditions (4.18) are fulfilled automatically [28]. This fact was proved earlier by another method in [29].

\subsection{Notations and preliminaries}

By $\mathbb{C}_{+}$, we denote the set of all $z \in \mathbb{C}$ with positive imaginary part and we set $\overline{\mathbb{C}}:=\mathbb{C} \cup\{\infty\}$ and $\overline{\mathbb{R}}:=\mathbb{R} \cup\{\infty\}$.

A complex function $m$ is called a Nevanlinna function if $m$ is holomorphic at least on $\mathbb{C} \backslash \mathbb{R}$ and satisfies the following two conditions:

$$
m(\bar{z})=\overline{m(z)} \quad \text { and } \quad \operatorname{Im} m(z) \geq 0, \quad \text { for all } \quad z \in \mathbb{C}_{+} .
$$

For information on Nevanlinna functions, we refer readers to [30] and [31, Chapter II].

All operators in this paper are closed densely defined linear operators. For such an operator $T$, we use the common notation $\rho(T), \operatorname{dom}(T), \operatorname{ran}(T)$ and $\operatorname{ker}(T)$ for the resolvent set, the domain, the range and the nullspace, respectively, of $T$. We define the extended spectrum $\widetilde{\sigma}(A)$ of $A$ by $\widetilde{\sigma}(A):=\sigma(A)$ if $A$ is bounded and $\tilde{\sigma}(A):=\sigma(A) \cup\{\infty\}$ if $A$ is unbounded and we set $\widetilde{\rho}(A):=\overline{\mathbb{C}} \backslash \widetilde{\sigma}(A)$.

\section{Definitizable operators in Krĕn spaces}

\subsection{Kreĭn spaces}

We recall standard notation and some basic results on Kreĭn spaces. For a complete exposition on the subject (and the proofs of the results below) see the books by Azizov and Iokhvidov [1] and Bognár [2]. A vector space $\mathcal{K}$ with a Hermitian sesquilinear form $[\cdot, \cdot]_{\mathcal{K}}$ is called a Krě̆ space if there exists a so-called fundamental decomposition

$$
\mathcal{K}=\mathcal{K}_{+}+\mathcal{K}_{-},
$$

such that $\left(\mathcal{K}_{+},[\cdot, \cdot]_{\mathcal{K}}\right)$ and $\left(\mathcal{K}_{-},-[\cdot, \cdot]_{\mathcal{K}}\right)$ are Hilbert spaces which are orthogonal to each other with respect to $[\cdot, \cdot]_{\mathcal{K}}$. Those two Hilbert spaces induce in a natural way a Hilbert space inner product $(\cdot, \cdot)$ and, hence, a Hilbert space topology on the Krein space $\mathcal{K}$. Observe that the indefinite metric $[\cdot, \cdot]_{\mathcal{K}}$ and the Hilbert space inner product $(\cdot, \cdot)$ of $\mathcal{K}$ are related by means of a fundamental symmetry, i.e. a unitary self-adjoint operator $J$ which satisfies

$$
(x, y)=[J x, y]_{\mathcal{K}} \quad \text { for } x, y \in \mathcal{K} .
$$

If $\mathcal{H}$ and $\mathcal{K}$ are Krein spaces and $T: \mathcal{H} \rightarrow \mathcal{K}$ a bounded operator, the adjoint operator $T^{+}$of $\mathrm{T}$ with respect to the Kreĭn spaces $\mathcal{H}$ and $\mathcal{K}$ is defined by:

$$
T^{+}:=J_{\mathcal{H}} T^{*} J_{\mathcal{K}}
$$

where $J_{\mathcal{H}}$ and $J_{\mathcal{K}}$ are the fundamental symmetries associated with $\mathcal{H}$ and $\mathcal{K}$, respectively; the operator $T^{+}$satisfies $[T x, y]_{\mathcal{K}}=\left[x, T^{+} y\right]_{\mathcal{K}}$ for all $x \in \mathcal{H}, y \in \mathcal{K}$. If $A$ is a densely defined operator in $\mathcal{K}$ then the adjoint $A^{+}$of $A$ with respect to $[\cdot, \cdot]_{\mathcal{K}}$ is defined analogously. In fact, if $J$ is a fundamental symmetry on $\left(\mathcal{K},[\cdot, \cdot]_{\mathcal{K}}\right)$ and $(\cdot, \cdot)$ is the corresponding Hilbert space inner product (2.1), then $A^{+}=J A^{*} J$. The operator $A^{+}$satisfies the following:

$$
[A x, y]_{\mathcal{K}}=\left[x, A^{+} y\right]_{\mathcal{K}} \quad \text { for all } x \in \operatorname{dom}(A), y \in \operatorname{dom}\left(A^{+}\right) .
$$

By analogy with the definitions in Hilbert spaces, $A$ is symmetric in $\left(\mathcal{K},[\cdot, \cdot]_{\mathcal{K}}\right)$ if $A^{+}$is an extension of $A$ and $A$ is self-adjoint in $\left(\mathcal{K},[\cdot, \cdot]_{\mathcal{K}}\right)$ if $A=A^{+}$. 
A densely defined operator $A$ is called nonnegative in $\left(\mathcal{K},[\cdot, \cdot]_{\mathcal{K}}\right)$ if $[A f, f]_{\mathcal{K}} \geq 0$ for all $f \in \operatorname{dom}(A)$. A nonnegative self-adjoint operator in a Kreĭn space can have an empty resolvent set; a specific example is given in [12, Section 1.2] and [2, Example VII.1.5]. But if a nonnegative self-adjoint operator in a Krein space also has a nonempty resolvent set, then it has real spectrum only.

An operator $A$ is called semibounded from below in the Kreĭn spaces $\left(\mathcal{K},[\cdot, \cdot]_{\mathcal{K}}\right)$, if there exists $\alpha \in \mathbb{R}$ such that:

$$
[A f, f]_{\mathcal{K}} \geq \alpha[f, f]_{\mathcal{K}}, \quad f \in \operatorname{dom}(A)
$$

\subsection{Definitizable operators}

In this section, we recall some facts on definitizable operators in Krein spaces. For an overview, we refer to [32], see also [33]. For this purpose, it is convenient to introduce in Definition 2.1 below the notion of sign-type spectra, cf. [34-37].

Let $A$ be a closed operator in a Kreĭn space $\left(\mathcal{K},[\cdot, \cdot]_{\mathcal{K}}\right)$. A point $\lambda_{0} \in \mathbb{C}$ is said to belong to the approximative point spectrum $\sigma_{a p}(A)$ of $A$ if there exists a sequence $\left(x_{n}\right)$ in $\operatorname{dom}(A)$ with $\left\|x_{n}\right\|=1, n=1,2, \ldots$, and $\left\|\left(A-\lambda_{0}\right) x_{n}\right\| \rightarrow 0$ if $n \rightarrow \infty$. For a self-adjoint operator $A$ in $\left(\mathcal{K},[\cdot, \cdot]_{\mathcal{K}}\right)$, all real spectral points of $A$ belong to $\sigma_{a p}(A)$ (see e.g. [2, Corollary VI.6.2]).

Definition 2.1. For a self-adjoint operator $A$ in $\left(\mathcal{K},[\cdot, \cdot]_{\mathcal{K}}\right)$ a point $\lambda_{0} \in \sigma(A)$ is called a spectral point of positive (negative) type of $A$ if $\lambda_{0} \in \sigma_{a p}(A)$ and for every sequence $\left(x_{n}\right)$ in $\operatorname{dom}(A)$ with $\left\|x_{n}\right\|=1, n=1,2, \ldots$, and $\left\|\left(A-\lambda_{0}\right) x_{n}\right\| \rightarrow 0$ for $n \rightarrow \infty$, we have:

$$
\left.\liminf _{n \rightarrow \infty}\left[x_{n}, x_{n}\right]_{\mathcal{K}}>0 \quad \text { (resp. } \limsup _{n \rightarrow \infty}\left[x_{n}, x_{n}\right]_{\mathcal{K}}<0\right) \text {. }
$$

The point $\infty$ is said to be a point of positive (negative) type of the extended spectrum of $A$ if $A$ is unbounded and for every sequence $\left(x_{n}\right)$ in $\operatorname{dom}(A)$ with $\lim _{n \rightarrow \infty}\left\|x_{n}\right\|=0$ and $\left\|A x_{n}\right\|=1, n=1,2, \ldots$, we have:

$$
\left.\liminf _{n \rightarrow \infty}\left[A x_{n}, A x_{n}\right]_{\mathcal{K}}>0 \quad \text { (resp. } \limsup _{n \rightarrow \infty}\left[A x_{n}, A x_{n}\right]_{\mathcal{K}}<0\right) \text {. }
$$

We denote the set of all points of $\widetilde{\sigma}(A)$ of positive (negative) type by $\sigma_{++}(A)$ (resp. $\sigma_{--}(A)$ ). Points from $\tilde{\sigma}(A)$ of neither positive nor negative type are called critical. In the following proposition, we collect some properties. For a proof, we refer to [34].

Proposition 2.2. $\quad$ (i) The sets $\sigma_{++}(A)$ and $\sigma_{--}(A)$ are contained in $\overline{\mathbb{R}}$.

(ii) The non-real spectrum of $A$ cannot accumulate to $\sigma_{++}(A) \cup \sigma_{--}(A)$.

(iii) The sets $\sigma_{++}(A)$ and $\sigma_{--}(A)$ are relatively open in $\tilde{\sigma}(A)$.

(iv) Let $\lambda_{0}$ be a point of $\sigma_{++}(A)\left(\sigma_{--}(A)\right.$, respectively). Then there exists an open vicinity $\mathcal{U}$ in $\overline{\mathbb{C}}$ of $\lambda_{0}$ and a number $M>0$ such that:

$$
\left\|(A-\lambda)^{-1}\right\| \leq \frac{M}{|\operatorname{Im} \lambda|} \text { for all } \lambda \in \mathcal{U} \backslash \overline{\mathbb{R}}
$$

We shall say that an open subset $\Delta$ of $\overline{\mathbb{R}}$ is of positive type (negative type) with respect to $A$ if:

$$
\left.\Delta \cap \tilde{\sigma}(A) \subset \sigma_{++}(A) \quad \text { (resp. } \Delta \cap \tilde{\sigma}(A) \subset \sigma_{--}(A)\right)
$$

An open set $\Delta$ of $\overline{\mathbb{R}}$ is called of definite type if $\Delta$ is of positive or of negative type with respect to $A$. If we relate Definition 2.1 to nonnegative operators in Kreĭn spaces (cf. Section 2.1), we obtain from the properties of the spectral function of a nonnegative operator in a Krein space, see, e.g., [1,32,38], and [34, Proposition 25] the following.

Proposition 2.3. Let $A$ be a nonnegative operator with $\rho(A) \neq \emptyset$ in a Krĕ̌n space $\left(\mathcal{K},[\cdot, \cdot]_{\mathcal{K}}\right)$. Then $c(A) \subset$ $\{0, \infty\}$ and

$$
\sigma(A) \cap(0, \infty) \subset \sigma_{++}(A) \subset \overline{\mathbb{R}} \backslash(-\infty, 0), \quad \sigma(A) \cap(-\infty, 0) \subset \sigma_{--}(A) \subset \overline{\mathbb{R}} \backslash(0, \infty) .
$$

In particular, we have:

$$
c(A)=\tilde{\sigma}(A) \backslash\left(\sigma_{++}(A) \cup \sigma_{--}(A)\right) .
$$

A generalization of the class of nonnegative operators in Kreĭn spaces is given by the class of definitizable operators. Recall, that a self-adjoint operator $A$ in a Krein space $\left(\mathcal{K},[\cdot, \cdot]_{\mathcal{K}}\right)$ is called definitizable if $\rho(A) \neq \emptyset$ and if there exists a rational function $p \neq 0$ having poles only in $\rho(A)$ such that $[p(A) x, x]_{\mathcal{K}} \geq 0$ for all $x \in \mathcal{K}$. Such a function $p$ is called definitizing function for $A$. Then the spectrum of $A$ is real or its non-real part consists 
of a finite number of points. Inspired by Proposition 2.3 we introduce the set of critical points of a definitizable operator $A$ via:

$$
c(A):=\tilde{\sigma}(A) \backslash\left(\sigma_{++}(A) \cup \sigma_{--}(A)\right) .
$$

It is known (cf. [32]) that $c(A)$ is contained in $\{t \in \mathbb{R}: p(t)=0\} \cup\{\infty\}$.

For the definitizable operator $A$, the spectral function $E(\Delta)$ can be introduced for every interval $\Delta$ such that the endpoints of $\Delta$ belong to intervals of definite type, see [32], [14]. We mention only that $E(\Delta)$ is defined and is a self-adjoint projection in $\left(\mathcal{K},[\cdot, \cdot]_{\mathcal{K}}\right)$ for every such interval. Moreover,

$$
\left(E(\Delta) \mathcal{K},[\cdot, \cdot]_{\mathcal{K}}\right) \text { is a Hilbert space whenever } \Delta \subset\{t \in \mathbb{R}: p(t)>0\}
$$

If a critical point $\alpha$ is the endpoint of two intervals $\left(\lambda_{1}, \alpha\right)$ and $\left(\alpha, \lambda_{2}\right)$ of the definite type, then the sequences $E\left(\left[\lambda_{1}, t\right]\right)$ and $E\left(\left[t, \lambda_{2}\right]\right)$ are monotone in $\left(\lambda_{1}, \alpha\right)$ and $\left(\alpha, \lambda_{2}\right)$, resp. The point $\alpha$ is called a regular critical point of $A$, if the limits

$$
\lim _{t \uparrow \alpha} E\left(\left[\lambda_{1}, t\right]\right) \quad \text { and } \quad \lim _{t \downarrow \alpha} E\left(\left[t, \lambda_{2}\right]\right)
$$

exist in the strong operator topology. A critical point of $A$ which is not regular is called singular critical point of $A$. The set of all singular critical points of $A$ is denoted by $c_{s}(A)$.

In Subsection 4.2, we essentially use the following resolvent criterion of K. Veselić $[25,26]$ for $\infty \notin c_{s}(A)$. We state a special case of this criterion as it has appeared in [27, Corollary 1.6].

Theorem 2.4. Let $A$ be a definitizable self-adjoint operator in a Krĕn space $(\mathcal{K},[\cdot, \cdot])$. Then:

(a) $\infty \notin c_{s}(A)$ if and only if there is $\eta_{0}>0$, such that the set of numbers:

$$
\int_{\eta_{0}}^{\eta} \operatorname{Re}\left[(A-i y)^{-1} f, f\right]_{\mathcal{K}} d y \quad\left(\eta \in\left(\eta_{0}, \infty\right)\right)
$$

is bounded for every $f \in \mathcal{K}$.

(b) Let $\xi_{0} \in \mathbb{R}$. Then $\xi_{0} \notin c_{s}(A)$ and $\operatorname{ker}\left(A-\xi_{0}\right)=\operatorname{ker}\left(A-\xi_{0}\right)^{2}$ if and only if there is $\eta_{0}>0$, such that the set of numbers:

$$
\int_{\eta}^{\eta_{0}} \operatorname{Re}\left[\left(A-\xi_{0}-i y\right)^{-1} f, f\right]_{\mathcal{K}} d y \quad\left(\eta \in\left(0, \eta_{0}\right)\right)
$$

is bounded for every $f \in \mathcal{K}$.

A characterization of definitizable operators via their sign-type spectrum together with some growth conditions for the resolvent is provided by the following theorem. Its proof follows from [35, Definition 4.4 and Theorem 4.7]).

Theorem 2.5. Let $A$ be a self-adjoint operator in the Krĕn space $\left(\mathcal{K},[\cdot, \cdot]_{\mathcal{K}}\right)$. Then $A$ is definitizable if and only if the following holds.

(i) The non-real spectrum $\sigma(A) \backslash \mathbb{R}$ consists of isolated points which are poles of the resolvent of $A$, and no point of $\overline{\mathbb{R}}$ is an accumulation point of the non-real spectrum $\sigma(A) \backslash \mathbb{R}$ of $A$.

(ii) There is an open vicinity $\mathcal{U}$ of $\overline{\mathbb{R}}$ in $\overline{\mathbb{C}}$ and numbers $m \geq 1, M>0$ with

$$
\left\|(A-\lambda)^{-1}\right\| \leq M(|\lambda|+1)^{2 m-2}|\operatorname{Im} \lambda|^{-m} \text { for all } \lambda \in \mathcal{U} \backslash \overline{\mathbb{R}} .
$$

(iii) Every point $\lambda \in \overline{\mathbb{R}}$ has an open connected vicinity $I_{\lambda}$ in $\overline{\mathbb{R}}$ such that both components of $I_{\lambda} \backslash\{\lambda\}$ are of definite type with respect to $A$.

\section{Locally definitizable operators and their direct sum}

\subsection{Locally definitizable operators in Kreĭn spaces}

In view of Theorem 2.5 , it is natural to introduce a local version of definitizability which will play an important role in the following. The next notion is due to P. Jonas, see [13,14], we mention also the overview in [39].

Definition 3.1. Let $\Omega$ be a domain in $\overline{\mathbb{C}}$ which is symmetric with respect to $\mathbb{R}$ such that $\Omega \cap \overline{\mathbb{R}} \neq \emptyset$ and the intersections with the open upper and lower half-plane are simply connected. Let $A$ be a self-adjoint operator in the Krein space $\left(\mathcal{K},[\cdot, \cdot]_{\mathcal{K}}\right)$. The operator $A$ is called definitizable over $\Omega$ if the following holds:

(i) The non-real spectrum in $\Omega$, i.e. $\sigma(A) \cap(\Omega \backslash \overline{\mathbb{R}})$, consists of isolated points which are poles of the resolvent of $A$, and no point of $\Omega \cap \overline{\mathbb{R}}$ is an accumulation point of the non-real spectrum $\sigma(A) \backslash \mathbb{R}$ of $A$. 
(ii) For every closed subset $\Delta$ of $\Omega \cap \overline{\mathbb{R}}$ there exist an open vicinity $\mathcal{U}$ of $\Delta$ in $\overline{\mathbb{C}}$ and numbers $m \geq 1, M>0$ such that

$$
\left\|(A-\lambda)^{-1}\right\| \leq M(|\lambda|+1)^{2 m-2}|\operatorname{Im} \lambda|^{-m} \quad \text { for all } \lambda \in \mathcal{U} \backslash \overline{\mathbb{R}} .
$$

(iii) Every point $\lambda \in \Omega \cap \overline{\mathbb{R}}$ has an open connected vicinity $I_{\lambda}$ in $\overline{\mathbb{R}}$ such that both components of $I_{\lambda} \backslash\{\lambda\}$ are of definite type with respect to $A$.

Let $A$ be definitizable over $\Omega$. Similar as in (2.3) we call a point $t \in \Omega \cap \overline{\mathbb{R}}$ a critical point of the operator $A$ if there is no open subset $\Delta$ of definite type with $t \in \Delta$. The set of critical points of $A$ is denoted by $c(A)$. As in Section 2.1, critical points admit a classification into singular and regular critical points: If for some $\lambda \in c(A) \backslash\{\infty\}$ the limits analogous to (2.5) exist, then $\lambda$ is called a regular critical point of $A$. If $\infty$ is a critical point of $A$ and the limits (2.5) exist in the strong operator topology for some $\lambda_{1}, \lambda_{2} \in \mathbb{R} \backslash\{0\}$, then $\infty$ is called regular critical point of $A$. A critical point of $A$ which is not regular is called singular critical point of $A$. The set of all singular critical points of $A$ is denoted by $c_{s}(A)$.

Theorem 2.4 has a counterpart for locally definitizable operators: Let $A$ be definitizable over a vicinity $\Omega$ of $\infty$. Then, $A$ admits an orthogonal decomposition into two operators: a definitizable one with spectrum in $\bar{\Delta}$ and a self-adjoint one with spectrum outside $\Delta$, where $\Delta(\subset \Omega)$ is a vicinity of $\infty$, for details we refer to [35, Theorem 4.8]. Then, the following theorem follows easily from this decomposition and Theorem 2.4:

Theorem 3.2. Let a self-adjoint operator $A$ in a Krĕn space $(\mathcal{K},[\cdot, \cdot])$ be locally definitizable over a neighborhood $\Omega$ of $\infty$. Then $\infty \notin c_{s}(A)$ if and only if there is $\eta_{0}>0$, such that the set of numbers:

$$
\int_{\eta_{0}}^{\eta} \operatorname{Re}\left[(A-i y)^{-1} f, f\right]_{\mathcal{K}} d y \quad\left(\eta \in\left(\eta_{0}, \infty\right)\right)
$$

is bounded for every $f \in \mathcal{K}$.

Similarly, if $\xi_{0} \in \mathbb{R}$ and $A$ is locally definitizable over a vicinity $\Omega$ of $\xi_{0}$, then $\xi_{0} \notin c_{s}(A)$ and $\operatorname{ker}\left(A-\xi_{0}\right)=$ $\operatorname{ker}\left(A-\xi_{0}\right)^{2}$ if and only if there is $\eta_{0}>0$, such that the set of numbers:

$$
\int_{\eta}^{\eta_{0}} \operatorname{Re}\left[\left(A-\xi_{0}-i y\right)^{-1} f, f\right]_{\mathcal{K}} d y \quad\left(\eta \in\left(0, \eta_{0}\right)\right)
$$

is bounded for every $f \in \mathcal{K}$.

Roughly speaking, the property of an operator to be definitizable or to be locally definitizable is stable under finite rank perturbations. This is made more precise in the following theorem which is taken from J. Behrndt [40, Theorem 2.2]:

Theorem 3.3. Let $A_{0}$ and $A_{1}$ be self-adjoint operators in a Krĕ̌n space $\left(\mathcal{K},[\cdot, \cdot]_{\mathcal{K}}\right)$ with $\rho\left(A_{0}\right) \cap \rho\left(A_{1}\right) \neq \emptyset$ and assume that for some $\lambda_{0} \in \rho\left(A_{0}\right) \cap \rho\left(A_{1}\right)$ the difference:

$$
\left(A_{0}-\lambda_{0}\right)^{-1}-\left(A_{1}-\lambda_{0}\right)^{-1}
$$

is a finite rank operator. Then, $A_{0}$ is definitizable over $\Omega$ if and only if $A_{1}$ is definitizable over $\Omega$.

Moreover, if $A_{0}$ is definitizable over $\Omega$ and $\delta \subset \Omega \cap \overline{\mathbb{R}}$ is an open interval with endpoint $\mu \in \Omega \cap \overline{\mathbb{R}}$ and the spectral points of $A_{0}$ in $\delta$ are only of positive type (negative type), then there exists an open interval $\delta^{\prime}, \delta^{\prime} \subset \delta$, with endpoint $\mu$ such that the spectral points of $A_{1}$ in $\delta^{\prime}$ are only of positive type (negative type, respectively).

Theorem 3.3 also holds for definitizable operators as the class of definitizable operators over $\overline{\mathbb{C}}$ coincides with the class of definitizable operators ( [35, Theorem 4.7]). For definitizable operators, this fact is already contained in [41].

\subsection{Local definitizability of the direct sum of two operators}

In this section, we characterize the definitizability of an operator which is the direct sum of two definitizable operators. For this, we provide the following definition:

Definition 3.4. We shall say that the sets $S_{1}$ and $S_{2}, S_{1}, S_{2} \subset \overline{\mathbb{R}}$, are separated by a finite number of points if there exists a finite ordered set $\left\{\alpha_{j}\right\}_{j=1}^{N}, N \in \mathbb{N}$ :

$$
-\infty=\alpha_{0}<\alpha_{1} \leq \cdots \leq \alpha_{N}<\alpha_{N+1}=+\infty
$$


such that one of the sets $S_{j}, j=1,2$, is a subset of $\bigcup_{k \text { is even }}\left[\alpha_{k}, \alpha_{k+1}\right]$ and the other one is a subset of $\bigcup_{k}\left[\alpha_{k}, \alpha_{k+1}\right]$. Here, we agree that 0 is even, $\left[\alpha_{0}, \alpha_{1}\right]$ stands for $\left(-\infty, \alpha_{1}\right] \cup\{\infty\}$ and $\left[\alpha_{N}, \alpha_{N+1}\right]$ for $k$ is odd $\left[\alpha_{N}, \infty\right) \cup\{\infty\}$.

The following theorem can be considered as a refinement of [42, Theorem 3.6]:

Theorem 3.5. Consider two operators $A$ and $B$ where $A$ is self-adjoint in the Krĕn space $\left(\mathcal{K}_{+},[\cdot, \cdot]_{\mathcal{K}_{+}}\right)$and $B$ in $\left(\mathcal{K}_{-},[\cdot, \cdot]_{\mathcal{K}_{-}}\right)$. Let the direct sum of the two Krĕn spaces:

$$
\mathcal{K}=\mathcal{K}_{+}[+] \mathcal{K}_{-},
$$

be endowed with the natural inner product:

$$
[f, g]_{\mathcal{K}}:=\left[P_{+} f, P_{+} g\right]_{\mathcal{K}_{+}}+\left[P_{-} f, P_{-} g\right]_{\mathcal{K}_{-}} \quad(f, g \in \mathcal{K})
$$

where $P_{ \pm}$are the orthogonal projections onto $\mathcal{K}_{ \pm}$. Then, the sum of the operators $A[+] B$ is self-adjoint in the direct sum of the Krĕn spaces $\mathcal{K}$ with the natural inner product from (3.1). We set the following:

$$
S_{+}:=\sigma_{++}(A) \cup \sigma_{++}(B) \text { and } S_{-}:=\sigma_{--}(A) \cup \sigma_{--}(B) .
$$

Then, $A[+] B$ is definitizable if and only if the operators $A$ and $B$ are definitizable and $S_{+}$and $S_{-}$are separated by a finite number of points.

Proof. The non-real-spectrum of $A[+] B$ coincides with the union of the non-real spectra of $A$ and of $B$. Therefore, if $A[+] B$ is definitizable, then item (i) of Theorem 2.5 holds for $A$ and for $B$. Conversely, if $A$ and $B$ are both definitizable, then (i) of Theorem 2.5 holds for $A[+] B$. Therefore, it is no restriction to assume that $A[+] B, A$, and $B$ have real spectrum only.

If $A[+] B$ is definitizable, then by the definition of the inner product in $\mathcal{K}=\mathcal{K}_{+}[+] \mathcal{K}_{-}$a definitizing function $p$ for $A[+] B$ is also a definitizing function for $A$ and for $B$. From (2.4), we deduce:

$$
\begin{array}{ll}
\{t \in \mathbb{R}: p(t)>0\} \subset \sigma_{++}(A) \cup \rho(A), & \{t \in \mathbb{R}: p(t)<0\} \subset \sigma_{--}(A) \cup \rho(A), \\
\{t \in \mathbb{R}: p(t)>0\} \subset \sigma_{++}(B) \cup \rho(B), & \{t \in \mathbb{R}: p(t)<0\} \subset \sigma_{--}(B) \cup \rho(B),
\end{array}
$$

and, hence, the zeros of $p$ are the points separating $S_{+}$and $S_{-}$, cf. Definition 3.4.

It remains to prove the converse. We assume that $S_{+}$and $S_{-}$are separated by the points $\left\{\alpha_{0}, \ldots, \alpha_{N+1}\right\}$, cf. Definition 3.4, then we have:

$$
S_{+} \cap S_{-} \subset\left\{\alpha_{0}, \ldots, \alpha_{N+1}\right\}
$$

Note that $S_{+}$and $c(A)$ may have a non-empty intersection (and the same applies to $S_{+} \cap c(B), S_{-} \cap c(A)$, and $S_{-} \cap c(B)$ ). Indeed, let $\lambda \in \sigma_{++}(B)$ (and, hence, $\lambda \in S_{+}$) such that $\lambda$ is an isolated spectral point of $A$ which belongs to $c(A)$. Then, $\lambda \in S_{+} \cap c(A)$ and, moreover as $\lambda \notin S_{-}$, we have in addition $\lambda \notin\left\{\alpha_{0}, \ldots, \alpha_{N+1}\right\}$.

We define:

$$
\Lambda:=\left\{\alpha_{0}, \ldots, \alpha_{N+1}\right\} \cup c(A) \cup c(B),
$$

and for $\lambda \in S_{+} \backslash \Lambda$, the following statements are true:

(i) $\lambda \in \sigma_{++}(A) \cup \sigma_{++}(B)$ (as $\lambda \in S_{+}$),

(ii) $\lambda \notin \sigma_{--}(A) \cup \sigma_{--}(B)$ (as $\lambda \notin S_{-}$),

(iii) $\lambda \notin c(A) \cup c(B)$ (as $\lambda \notin \Lambda)$.

Thus, by (2.2) applied to both $A$ and $B$, we obtain:

$$
\lambda \in \sigma_{++}(A) \cup \widetilde{\rho}(A) \quad \text { and } \quad \lambda \in \sigma_{++}(B) \cup \widetilde{\rho}(B) .
$$

This implies:

$$
\lambda \in \sigma_{++}(A[+] B),
$$

and we obtain:

and with similar arguments:

$$
S_{+} \backslash \Lambda \subset \sigma_{++}(A[+] B),
$$

$$
S_{-} \backslash \Lambda \subset \sigma_{--}(A[+] B) .
$$


From (2.2), we conclude:

$$
\begin{aligned}
\widetilde{\sigma}(A[+] B) & =\widetilde{\sigma}(A) \cup \widetilde{\sigma}(B) \\
& =\sigma_{++}(A) \cup c(A) \cup \sigma_{--}(A) \cup \sigma_{++}(B) \cup c(B) \cup \sigma_{--}(B) \\
& =S_{+} \cup c(A) \cup c(B) \cup S_{-} \subset S_{+} \cup S_{-} \cup \Lambda .
\end{aligned}
$$

Obviously, for the operator $A[+] B$ the statements (i) and (ii) from Theorem 2.5 are satisfied as $A$ and $B$ are definitizable operators. It remains to show (iii). Clearly, for $\lambda \in \overline{\mathbb{C}} \backslash \widetilde{\sigma}(A[+] B)$ (iii) in Theorem 2.5 is satisfied. Let $\lambda \in \widetilde{\sigma}(A[+] B)$. If $\lambda \in\left(S_{+} \cup S_{-}\right) \backslash \Lambda$ we deduce from (3.2) and (3.3) that either $\lambda \in \sigma_{++}(A[+] B)$ or $\lambda \in$ $\sigma_{--}(A[+] B)$. As the sets $\sigma_{++}(A[+] B)$ and $\sigma_{--}(A[+] B)$ are relatively open in $\widetilde{\sigma}(A[+] B)$ (cf. Proposition 2.2), (iii) follows. By (3.4), it remains to consider $\lambda \in \Lambda$. For $\lambda \in\left\{\alpha_{0}, \ldots, \alpha_{N+1}\right\}$ (iii) follows from (3.2) and (3.3). Therefore, consider $\lambda \in c(A) \cup c(B)$. It is sufficient to consider $\lambda \in c(A) \backslash\left\{\alpha_{0}, \ldots, \alpha_{N+1}\right\}$. It follows from the definition of the points $\left\{\alpha_{0}, \ldots, \alpha_{N+1}\right\}$ and the fact that $\lambda \notin\left\{\alpha_{0}, \ldots, \alpha_{N+1}\right\}$ that there exists open connected vicinities $I_{\lambda}, J_{\lambda}$ in $\overline{\mathbb{R}}$ of $\lambda$ with:

$$
\left(I_{\lambda} \backslash\{\lambda\}\right) \cap \tilde{\sigma}(A) \subset \sigma_{++}(A) \quad \text { and } \quad\left(J_{\lambda} \backslash\{\lambda\}\right) \cap \widetilde{\sigma}(B) \subset \sigma_{++}(B)
$$

or

$$
\left(I_{\lambda} \backslash\{\lambda\}\right) \cap \widetilde{\sigma}(A) \subset \sigma_{--}(A) \quad \text { and } \quad\left(J_{\lambda} \backslash\{\lambda\}\right) \cap \widetilde{\sigma}(B) \subset \sigma_{--}(B) .
$$

This shows $\left(I_{\lambda} \cap J_{\lambda} \backslash\{\lambda\}\right) \cap \widetilde{\sigma}(A[+] B)$ is a subset of $\sigma_{++}(A[+] B)$ or of $\sigma_{--}(A[+] B)$ and (iii) follows.

Corollary 3.6. Let $A_{+}$and $A_{-}$be self-adjoint and semibounded from below in the Krein spaces $\left(\mathcal{K}_{+},[\cdot, \cdot]_{\mathcal{K}_{+}}\right)$ and $\left(\mathcal{K}_{-},[\cdot, \cdot]_{\mathcal{K}_{-}}\right)$, respectively:

$$
\left[A_{ \pm} f_{ \pm}, f_{ \pm}\right]_{\mathcal{K}_{ \pm}} \geq \alpha_{ \pm}\left[f_{ \pm}, f_{ \pm}\right]_{\mathcal{K}_{ \pm}}, \quad f_{ \pm} \in \operatorname{dom}\left(A_{ \pm}\right)
$$

for some $\alpha_{ \pm} \in \mathbb{R}$. Let $\rho\left(A_{+}\right) \neq \emptyset, \rho\left(A_{-}\right) \neq \emptyset$. Then, their direct sum $A_{+}[+] A_{-}$is definitizable over:

$$
\Omega:=\overline{\mathbb{C}} \backslash\left[\min \left\{\alpha_{+}, \alpha_{-}\right\}, \max \left\{\alpha_{+}, \alpha_{-}\right\}\right],
$$

in the direct sum of the Krěn spaces $\mathcal{K}=\mathcal{K}_{+}[+] \mathcal{K}_{-}$. In particular, $A_{+}[+] A_{-}$is definitizable if and only if the sets $S_{+}$and $S_{-}$from Theorem 3.5 are separated by a finite number of points.

This is fulfilled in the following special cases:

(I) $\alpha_{-}=\alpha_{+}$.

(II) $\alpha_{-}<\alpha_{+}$and either $\sigma\left(A_{+}\right) \cap\left(\alpha_{-}, \alpha_{+}\right)$is finite or $\sigma\left(A_{-}\right) \cap\left(\alpha_{-}, \alpha_{+}\right)$is finite.

(III) $\alpha_{+}<\alpha_{-}$and either $\sigma\left(A_{+}\right) \cap\left(\alpha_{+}, \alpha_{-}\right)$is finite or $\sigma\left(A_{-}\right) \cap\left(\alpha_{+}, \alpha_{-}\right)$is finite.

Proof. The assumptions on $A_{ \pm}$imply that $A_{+}-\alpha_{+}$and $A_{-}-\alpha_{-}$are nonnegative operators and, hence, $A_{ \pm}$are definitizable operators. Then, with Proposition 2.3, we see that:

$$
\left(\alpha_{ \pm}, \infty\right) \cap \sigma\left(A_{ \pm}\right) \subset \sigma_{++}\left(A_{ \pm}\right) \quad \text { and } \quad\left(-\infty, \alpha_{ \pm}\right) \cap \sigma\left(A_{ \pm}\right) \subset \sigma_{--}\left(A_{ \pm}\right)
$$

and properties (i)-(iii) from Definition 3.1 for the operator $A_{+}[+] A_{-}$and $\Omega$ as in (3.6) are easily shown, cf. Proposition 2.2. Therefore, $A_{+}[+] A_{-}$is definitizable over $\Omega$.

The statements on the definitizability of the operator $A_{+}[+] A_{-}$now follow directly from (3.7) and Theorem 3.5.

\section{Coupling of definitizable operators in Kreĭn spaces}

\subsection{Boundary triples and Weyl functions of symmetric operators}

Starting from this section, we will denote by $A$ a closed densely defined symmetric operator in a Krě̆n space $\left(\mathcal{K},[\cdot, \cdot]_{\mathcal{K}}\right)$. Let $\widehat{\rho}(A)$ denote the set of points of regular type of $A$, see [43], and let $\mathfrak{N}_{z}$ denote the defect subspace of the operator $A$ :

$$
\mathfrak{N}_{z}:=\mathcal{H} \ominus \operatorname{ran}(A-\bar{z})=\operatorname{ker}\left(A^{+}-z\right), \quad z \in \widehat{\rho}(A) .
$$

In what follows, we assume that the operator $A$ admits a self-adjoint extension $\widetilde{A}$ in $\left(\mathcal{K},[\cdot, \cdot]_{\mathcal{K}}\right)$ with a nonempty resolvent set $\rho(\widetilde{A})$. Then, for all $z \in \rho(\widetilde{A})$, we have:

$$
\operatorname{dom}\left(A^{+}\right)=\operatorname{dom}(\widetilde{A})+\mathfrak{N}_{z} \quad \text { direct sum in } \quad \mathcal{H} .
$$

This implies, in particular, that the $\operatorname{dimension} \operatorname{dim}\left(\mathfrak{N}_{z}\right)$ is constant for all $z \in \rho(\widetilde{A})$.

Definition 4.1. Let $\Gamma_{0}$ and $\Gamma_{1}$ be linear mappings from $\operatorname{dom}\left(A^{+}\right)$to $\mathbb{C}^{d}$ such that: 
(i) the mapping $\Gamma: f \rightarrow\left\{\Gamma_{0} f, \Gamma_{1} f\right\}$ from $\operatorname{dom}\left(A^{+}\right)$to $\mathbb{C}^{2 d}$ is surjective;

(ii) the abstract Green's identity:

$$
\left[A^{+} f, g\right]_{\mathcal{K}}-\left[f, A^{+} g\right]_{\mathcal{K}}=\left(\Gamma_{0} g\right)^{*}\left(\Gamma_{1} f\right)-\left(\Gamma_{1} g\right)^{*}\left(\Gamma_{0} f\right)
$$

holds for all $f, g \in \operatorname{dom}\left(A^{+}\right)$.

Then, the triplet $\Pi=\left\{\mathbb{C}^{d}, \Gamma_{0}, \Gamma_{1}\right\}$ is said to be a boundary triple for $A^{+}$, see $[19,44,45$, Sect.3.1.4] for a much more general setting.

It follows from (4.2) that the extensions $A_{0}, A_{1}$ of $A$ defined as restrictions of $A^{+}$to the domains:

$$
\operatorname{dom}\left(A_{0}\right):=\operatorname{ker}\left(\Gamma_{0}\right) \quad \text { and } \quad \operatorname{dom}\left(A_{1}\right):=\operatorname{ker}\left(\Gamma_{1}\right)
$$

are self-adjoint extensions of $A$.

If $A$ has a self-adjoint extension $\widetilde{A}$, with $\rho(\widetilde{A}) \neq \emptyset$, then the operator $A^{+}$admits a boundary triple $\left\{\mathbb{C}^{d}, \Gamma_{0}, \Gamma_{1}\right\}$, such that $A_{0}=\widetilde{A}$ and $d=\operatorname{dim} \mathfrak{N}_{z}\left(z \in \rho\left(A_{0}\right)\right)$. In this case, for every $z \in \rho\left(A_{0}\right)$, the decomposition (4.1) holds with $\widetilde{A}=A_{0}$ and the mapping $\left.\Gamma_{0}\right|_{\mathfrak{N}_{z}}$ is invertible for every $z \in \rho\left(A_{0}\right)$. Therefore, the operator-function:

$$
\gamma(z):=\left(\left.\Gamma_{0}\right|_{\mathfrak{N}_{z}}\right)^{-1},
$$

is well defined and takes values in the set of bounded operators from $\mathbb{C}^{d}$ to $\mathfrak{N}_{z}$. The operator-function $\gamma(z)$ is called the $\gamma$-field of $A$, associated with the boundary triple $\Pi$. Notice, that $\gamma(z)$ satisfies the equality:

$$
\gamma(z)=\left(A_{0}-z_{0}\right)\left(A_{0}-z\right)^{-1} \gamma\left(z_{0}\right) \quad\left(z, z_{0} \in \rho\left(A_{0}\right)\right) .
$$

Definition 4.2. The matrix valued function $M: \rho\left(A_{0}\right) \rightarrow \mathbb{C}^{d \times d}$ is defined by the equality:

$$
M(z) \Gamma_{0} f_{z}=\Gamma_{1} f_{z}, \quad f_{z} \in \mathfrak{N}_{z}, z \in \rho\left(A_{0}\right) .
$$

The matrix valued function $M$ is called the Weyl function of $A$ corresponding to the boundary triple $\Pi=$ $\left\{\mathbb{C}^{d}, \Gamma_{0}, \Gamma_{1}\right\}$.

Clearly,

$$
M(z)=\Gamma_{1} \gamma(z), \quad z \in \rho\left(A_{0}\right),
$$

and hence $M(z)$ is well defined and takes values in $\mathbb{C}^{d \times d}$. It follows from the identity that the Weyl function $M(\lambda)$ satisfies the identities:

$$
M(z)-M(w)^{*}=(z-\bar{w}) \gamma(w)^{+} \gamma(z), \quad z, w \in \rho\left(A_{0}\right) .
$$

With $w=\bar{z}$ the identity (4.7) yields that the Weyl function $M$ satisfies the symmetry condition:

$$
M(\bar{z})^{*}=M(z) \text { for all } z \in \rho\left(A_{0}\right) .
$$

The identity (4.7) was used in [46] as a definition of the $Q$-function. In the case when $\left(\mathcal{K},[\cdot, \cdot]_{\mathcal{K}}\right)$ is a Hilbert space, it follows from (4.7) and (4.8) that $M$ is a Nevanlinna matrix valued function cf. (1.2).

In what follows, the function:

$$
\widehat{f}(z):=[f, \gamma(\bar{z})]_{\mathcal{K}} \quad\left(f \in \mathcal{K}, z \in \rho\left(A_{0}\right)\right)
$$

is called the generalized Fourier transform of $f$ associated with the boundary triple $\left\{\mathbb{C}, \Gamma_{0}, \Gamma_{1}\right\}$. A motivation for this name is hidden in the fact, that the mapping $f \mapsto \widehat{f}$ is a unitary mapping from $\mathcal{K}$ to a reproducing kernel Kreĭn space with the kernel $\frac{M(z)-M(\bar{w})}{z-\bar{w}}$ (see [28] for the Hilbert space case).

Proposition 4.3. [44-46] Let $A_{1}$ be the self-adjoint extension of $A$ with the domain defined in (4.3) and let $d=1$. For every $z \in \rho\left(A_{0}\right)$, the following equivalence holds:

$$
z \in \rho\left(A_{1}\right) \Longleftrightarrow M(z) \neq 0,
$$

and the resolvent of $A_{1}$ can be found by the formula:

$$
\left(A_{1}-z\right)^{-1} f=\left(A_{0}-z\right)^{-1} f-\frac{\widehat{f}(z)}{M(z)} \gamma(z),
$$

for all $f \in \mathcal{H}$ and all $z \in \rho\left(A_{0}\right) \cap \rho\left(A_{1}\right)$. 


\subsection{Construction of the coupling of two self-adjoint operators in a Kreinn space}

In this section, we consider two Kreĭn spaces $\left(\mathcal{K}_{+},[\cdot, \cdot]_{\mathcal{K}_{+}}\right)$and $\left(\mathcal{K}_{-},[\cdot, \cdot]_{\mathcal{K}_{-}}\right)$. Let their direct sum:

$$
\mathcal{K}=\mathcal{K}_{+}[+] \mathcal{K}_{-},
$$

be endowed with the natural inner product (3.1). Consider two closed symmetric densely defined operators $A_{+}$and $A_{-}$with defect numbers $(1,1)$ acting in the Kreln spaces $\left(\mathcal{K}_{+},[\cdot, \cdot]_{\mathcal{K}_{+}}\right)$and $\left(\mathcal{K}_{-},[\cdot, \cdot]_{\mathcal{K}_{-}}\right)$. Let $\left\{\mathbb{C}, \Gamma_{0}^{ \pm}, \Gamma_{1}^{ \pm}\right\}$be a boundary triple for $A_{ \pm}^{+}$. Let $M_{ \pm}$be the corresponding Weyl function and $\gamma_{A_{ \pm}}$the $\gamma$-field. By $A_{ \pm, 0}$, we denote the self-adjoint extension of $A_{ \pm}$which is defined on:

$$
\operatorname{dom}\left(A_{ \pm, 0}\right)=\operatorname{ker}\left(\Gamma_{0}^{ \pm}\right) \quad \text { by } \quad A_{ \pm, 0}=\left.A_{ \pm}^{+}\right|_{\operatorname{ker}\left(\Gamma_{0}^{ \pm}\right)},
$$

and assume that $\rho\left(A_{+, 0}\right) \cap \rho\left(A_{-, 0}\right) \neq \emptyset$. Then, the functions $M_{ \pm}$are defined and holomorphic on $\rho\left(A_{ \pm, 0}\right)$.

The following theorem is the indefinite version of a result from [47] (see also [28]).

Theorem 4.4. Under the general assumptions of this subsection we have:

(a) The linear operator $A$ defined as the restriction of $A_{+}^{+}[+] A_{-}^{+}$to the domain:

$$
\operatorname{dom}(A)=\left\{\left(\begin{array}{l}
f_{+} \\
f_{-}
\end{array}\right): \begin{array}{l}
\Gamma_{0}^{+}\left(f_{+}\right)=\Gamma_{0}^{-}\left(f_{-}\right)=0, \\
\Gamma_{1}^{+}\left(f_{+}\right)+\Gamma_{1}^{-}\left(f_{-}\right)=0,
\end{array} \quad f_{ \pm} \in \operatorname{dom}\left(A_{ \pm}^{+}\right)\right\}
$$

is closed, densely defined and symmetric with defect numbers $(1,1)$ in the Krĕn space $\mathcal{K}$.

(b) The adjoint $A^{+}$of $A$ is the restriction of $A_{+}^{+}[+] A_{-}^{+}$to the domain:

$$
\operatorname{dom}\left(A^{+}\right)=\left\{\left(\begin{array}{l}
f_{+} \\
f_{-}
\end{array}\right): \Gamma_{0}^{+}\left(f_{+}\right)-\Gamma_{0}^{-}\left(f_{-}\right)=0, f_{ \pm} \in \operatorname{dom}\left(A_{ \pm}^{+}\right)\right\} .
$$

(c) A boundary triple $\left\{\mathbb{C}, \Gamma_{0}, \Gamma_{1}\right\}$ for $A^{+}$is given by:

$$
\Gamma_{0} f=\Gamma_{0}^{+} f_{+}, \quad \Gamma_{1} f=\Gamma_{1}^{+} f_{+}+\Gamma_{1}^{-} f_{-}, \quad f=\left(\begin{array}{l}
f_{+} \\
f_{-}
\end{array}\right) \in \operatorname{dom}\left(A^{+}\right) .
$$

(d) The Weyl function $M(z)$ and the $\gamma$-field of $A$ relative to the boundary triple $\left\{\mathbb{C}, \Gamma_{0}, \Gamma_{1}\right\}$ are given by:

$$
M(z)=M_{+}(z)+M_{-}(z), \quad \gamma(z)=\left(\begin{array}{l}
\gamma_{A_{+}}(z) \\
\gamma_{A_{-}}(z)
\end{array}\right) \quad z \in \mathbb{C} \backslash \mathbb{R} .
$$

(e) The self-adjoint extension $A_{1}$ of $A$ such that $\operatorname{dom}\left(A_{1}\right)=\operatorname{ker}\left(\Gamma_{1}\right)$ coincides with the restriction of $A_{+}^{+}[+] A_{-}^{+}$to the domain:

$$
\operatorname{dom}\left(A_{1}\right)=\left\{\left(\begin{array}{l}
f_{+} \\
f_{-}
\end{array}\right): \begin{array}{l}
\Gamma_{0}^{+}\left(f_{+}\right)-\Gamma_{0}^{-}\left(f_{-}\right)=0, \\
\Gamma_{1}^{+}\left(f_{+}\right)+\Gamma_{1}^{-}\left(f_{-}\right)=0,
\end{array} f_{ \pm} \in \operatorname{dom}\left(A_{ \pm}^{+}\right)\right\}
$$

and is called a coupling of $A_{+}$and $A_{-}$relative to the boundary triples $\left\{\mathbb{C}, \Gamma_{0}^{+}, \Gamma_{1}^{+}\right\}$and $\left\{\mathbb{C}, \Gamma_{0}^{-}, \Gamma_{1}^{-}\right\}$.

(f) The self-adjoint extension $A_{0}$ of $A$ coincides with the direct sum $A_{+, 0}[+] A_{-, 0}$ and $\rho\left(A_{0}\right)=\rho\left(A_{+, 0}\right) \cap$ $\rho\left(A_{-, 0}\right) \neq \emptyset$.

(g) The resolvent set $\rho\left(A_{1}\right)$ is nonempty if and only if

$$
M_{+}+M_{-} \not \equiv 0 \text {. }
$$

For every $z \in \rho\left(A_{1}\right) \cap \rho\left(A_{0}\right)$ and $f=\left(\begin{array}{c}f_{+} \\ f_{-}\end{array}\right) \in \mathcal{K}=\mathcal{K}_{+}[+] \mathcal{K}_{-}$, the resolvent of $A_{1}$ is given by:

$$
\left(A_{1}-z\right)^{-1} f=\left(A_{0}-z\right)^{-1} f-\frac{\widehat{f}_{A_{+}}(z)+\widehat{f}_{A_{-}}(z)}{M_{+}(z)+M_{-}(z)} \gamma(z)
$$

where:

$$
\widehat{f}_{A_{+}}(z):=\left[f_{+}, \gamma_{A_{+}}(\bar{z})\right]_{\mathcal{K}_{+}}, \quad \widehat{f}_{A_{-}}(z):=\left[f_{-}, \gamma_{A_{-}}(\bar{z})\right]_{\mathcal{K}_{-}} .
$$


Proof. (a)-(c) Since $\left\{\mathbb{C}, \Gamma_{0}^{ \pm}, \Gamma_{1}^{ \pm}\right\}$is a boundary triple for $A_{ \pm}^{+}$, it follows from (4.2) that for all $f_{ \pm} \in \operatorname{dom}\left(A_{ \pm}^{+}\right)$:

$$
\begin{aligned}
{\left[A_{+}^{+} f_{+}, g_{+}\right]_{\mathcal{K}_{+}} } & -\left[f_{+}, A_{+}^{+} g_{+}\right]_{\mathcal{K}_{-}}+\left[A_{-}^{+} f_{-}, g_{-}\right] \mathcal{K}_{-}-\left[f_{-}, A_{-}^{+} g_{-}\right] \mathcal{K}_{-} \\
& =\overline{\left(\Gamma_{0}^{+} g_{+}\right)}\left(\Gamma_{1}^{+} f_{+}\right)-\overline{\left(\Gamma_{1}^{+} g_{+}\right)}\left(\Gamma_{0}^{+} f_{+}\right)+\overline{\left(\Gamma_{0}^{-} g_{-}\right)}\left(\Gamma_{1}^{-} f_{-}\right)-\overline{\left(\Gamma_{1}^{-} g_{-}\right)}\left(\Gamma_{0}^{-} f_{-}\right) .
\end{aligned}
$$

We denote by $T$ the restriction of $A_{+}^{+}[+] A_{-}^{+}$to the set of the right hand side of (4.10).

If

$$
f=\left(\begin{array}{l}
f_{+} \\
f_{-}
\end{array}\right), g=\left(\begin{array}{l}
g_{+} \\
g_{-}
\end{array}\right) \in \operatorname{dom}(T) \quad \text { then } \quad \Gamma_{0}^{+} f_{+}=\Gamma_{0}^{-} f_{-} \quad \text { and } \quad \Gamma_{0}^{+} g_{+}=\Gamma_{0}^{-} g_{-},
$$

and hence, one obtains from (4.16):

$$
[T f, g]_{\mathcal{K}}-[f, T g]_{\mathcal{K}}=\overline{\Gamma_{0}^{+} g_{+}}\left(\Gamma_{1}^{+} f_{+}+\Gamma_{1}^{-} f_{-}\right)-\overline{\left(\Gamma_{1}^{+} g_{+}+\Gamma_{1}^{-} g_{-}\right)} \Gamma_{0}^{+} f_{+} .
$$

Now, it follows from (4.17) that $A$ is a closed, densely defined and symmetric operator in the Krein space $\mathcal{K}$, $T=A^{+}$and a boundary triple for $A^{+}$can be chosen in the form (4.11).

(d) The formulas for $M$ and $\gamma$ are implied by (4.11), (4.4) and (4.5).

(e) \& (f) As $\left\{\mathbb{C}, \Gamma_{0}, \Gamma_{1}\right\}$ is a boundary triple for $A^{+}$, the extension $A_{1}$ with $\operatorname{dom}\left(A_{1}\right)=\operatorname{ker}\left(\Gamma_{1}\right)$ being a restriction of $A_{+}^{+}[+] A_{-}^{+}$. The formula (4.13) for the domain follows from $A_{1} \subset A^{+}$(see (4.10)) and $\operatorname{dom}\left(A_{1}\right)=$ $\operatorname{ker}\left(\Gamma_{1}\right)$. The statement $(\mathrm{f})$ is immediate from (4.10) and (4.11).

(g) The statement (g) is implied by (4.12) and Proposition 4.3.

Remark 4.5. The construction in Theorem 4.4 shows that the coupling of two self-adjoint operators $A_{+, 0}$ and $A_{-, 0}$ is not uniquely defined. Namely, let the boundary triples $\Pi^{-}=\left\{\mathbb{C}, \Gamma_{0}^{-}, \Gamma_{1}^{-}\right\}$and $\widetilde{\Pi}^{-}=\left\{\mathbb{C}, \widetilde{\Gamma}_{0}^{-}, \widetilde{\Gamma}_{1}^{-}\right\}$be related by

$$
\widetilde{\Gamma}_{0}^{-}=c \Gamma_{0}^{-}, \quad \widetilde{\Gamma}_{1}^{-}=\bar{c}^{-1} \Gamma_{1}^{-},
$$

for some non-zero $c \in \mathbb{C}, c \neq 1$. Then, the extension $\widetilde{A}_{1}$ defined as the restriction of $A_{+}^{+}[+] A_{-}^{+}$to the domain:

$$
\operatorname{dom}\left(\widetilde{A}_{1}\right)=\left\{\left(\begin{array}{l}
f_{+} \\
f_{-}
\end{array}\right): \begin{array}{l}
\Gamma_{0}^{+}\left(f_{+}\right)-c \Gamma_{0}^{-}\left(f_{-}\right)=0, \\
\Gamma_{1}^{+}\left(f_{+}\right)+\bar{c}^{-1} \Gamma_{1}^{-}\left(f_{-}\right)=0,
\end{array} f_{ \pm} \in \operatorname{dom}\left(A_{ \pm}^{+}\right)\right\},
$$

is also a coupling of $A_{-}$and $A_{+}$with $\widetilde{A}_{1} \neq A_{1}$.

However, when the boundary triples $\left\{\mathbb{C}, \Gamma_{0}^{ \pm}, \Gamma_{1}^{ \pm}\right\}$are fixed, then the coupling $A_{1}$ of the operators $A_{ \pm}$is uniquely defined by the formula (4.13) and is called the coupling of the operators $A_{ \pm, 0}$ relative to the boundary triples $\left\{\mathbb{C}, \Gamma_{0}^{ \pm}, \Gamma_{1}^{ \pm}\right\}$.

Let us suppose that the operators $A_{ \pm, 0}$ are semibounded from below, that is there exists $\alpha_{ \pm} \in \mathbb{R}$ such that (3.5) holds. Then, the results of Section 3.2 allow us to show that the coupling $A_{1}$ of the operators $A_{+, 0}$ and $A_{-, 0}$ is at least locally definitizable in a vicinity of $\infty$. In the next theorem, sufficient conditions for regularity of the critical point $\infty$ are given.

Theorem 4.6. Under the general assumptions of this subsection, we assume that the operators $A_{ \pm, 0}$, the $\gamma-$ fields $\gamma_{ \pm}$and the Weyl functions $M_{ \pm}$satisfy the following assumptions:

(A1) The operators $A_{ \pm, 0}$ are semibounded from below, $\rho\left(A_{ \pm, 0}\right) \neq \emptyset$, and

$$
\infty \notin c_{s}\left(A_{ \pm, 0}\right) .
$$

(A2) $(w(z):=)\left|M_{+}(z)+M_{-}(z)\right| \not \equiv 0$ on $\rho\left(A_{+, 0}\right) \cap \rho\left(A_{-, 0}\right)$.

(A3) There is $y_{1}>0$, such that for all $f_{A_{ \pm}} \in \mathcal{K}_{ \pm}$:

$$
\int_{y_{1}}^{\infty} \frac{\left|\widehat{f}_{A_{ \pm}}(i y)\right|^{2}}{w(i y)} d y<\infty, \quad \int_{y_{1}}^{\infty} \frac{\left|\widehat{f}_{A_{ \pm}}(-i y)\right|^{2}}{w(i y)} d y<\infty
$$

where the generalized Fourier transforms $\widehat{f}_{A_{+}}$and $\widehat{f}_{A_{-}}$are defined by (4.15).

Then, the coupling $A_{1}$ of the operators $A_{+, 0}$ and $A_{-, 0}$ is definitizable over $\Omega$, where $\Omega$ is as in (3.6). Moreover, we have:

$$
\infty \notin c_{s}\left(A_{1}\right) \text {. }
$$


Proof. By Corollary 3.6, the operator $A_{0}=A_{+, 0}[+] A_{-, 0}$ is definitizable over $\Omega$. In view of Theorem 4.4, the assumption (A2) yields $\rho\left(A_{1}\right) \neq \emptyset$. Since the operator $A_{1}$ is a two-dimensional perturbation of $A_{0}$, by Theorem 3.3, the operator $A_{1}$ is also definitizable over $\Omega$.

Clearly, $\infty \notin c_{s}\left(A_{0}\right)$ and it follows from Theorem 3.2 that there is $y_{2}>y_{1}>0$, such that:

$$
\int_{y_{2}}^{\infty}\left|\operatorname{Re}\left[\left(A_{0}-i y\right)^{-1} f, f\right]_{\mathcal{K}}\right| d y<\infty \text { for all } f \in \mathcal{K} \text {. }
$$

Let us set:

We show:

$$
\mathcal{A}(f, i y):=\frac{\left(\widehat{f}_{A_{+}}(i y)+\widehat{f}_{A_{-}}(i y)\right) \overline{\left(\widehat{f}_{A_{+}}(-i y)+\widehat{f}_{A_{-}}(-i y)\right)}}{M_{+}(i y)+M_{-}(i y)} .
$$

$$
\int_{y_{2}}^{\infty}|\mathcal{A}(f, i y)| d y<\infty \text { for all } f \in \mathcal{K} .
$$

It follows from (A3) that for every $f_{A_{ \pm}} \in \mathcal{K}_{ \pm}$

$$
\int_{y_{2}}^{\infty}\left|\widehat{f}_{A_{ \pm}}(i y) \widehat{f}_{A_{ \pm}}(-i y)\right| \frac{d y}{w(i y)} \leq\left(\int_{y_{2}}^{\infty}\left|\widehat{f}_{A_{ \pm}}(i y)\right|^{2} \frac{d y}{w(i y)}\right)^{1 / 2}\left(\int_{y_{2}}^{\infty}\left|\widehat{f}_{A_{ \pm}}(-i y)\right|^{2} \frac{d y}{w(i y)}\right)^{1 / 2}<\infty .
$$

Similarly, one obtains for all $f_{A_{ \pm}} \in \mathcal{K}_{ \pm}$:

$$
\int_{y_{2}}^{\infty}\left|\widehat{f}_{A_{+}}(i y) \widehat{f}_{A_{-}}(-i y)\right| \frac{d y}{w(i y)}<\infty .
$$

Combining (4.20) and (4.21), one obtains from (4.19) for all $f \in \mathcal{K}$

$$
\int_{y_{2}}^{\infty}|\mathcal{A}(f, i y)| d y=\int_{y_{2}}^{\infty}\left|\frac{\left(\widehat{f}_{A_{+}}(i y)+\widehat{f}_{A_{-}}(i y)\right) \overline{\left(\widehat{f}_{A_{+}}(-i y)+\widehat{f}_{A_{-}}(-i y)\right)}}{M_{+}(i y)+M_{-}(i y)}\right| d y<\infty .
$$

Now the statement $\infty \notin c_{s}\left(A_{1}\right)$ is implied by Theorem 2.4 and (4.14).

Theorem 4.7. Under the assumptions of this subsection we assume that the operators $A_{ \pm, 0}$, the $\gamma-$ fields $\gamma_{ \pm}$and the Weyl functions $M_{ \pm}$satisfy the following conditions:

$\left(A 1^{\prime}\right)$ The operators $A_{ \pm, 0}$ are semibounded from below, $\rho\left(A_{ \pm, 0}\right) \neq \emptyset$, one of the conditions (i), (ii) or (iii) of Corollary 3.6 holds, and $\alpha:=\min \left\{\alpha_{-}, \alpha_{+}\right\}$satisfies:

$$
\alpha \notin c_{s}\left(A_{ \pm, 0}\right) .
$$

$\left(A 2^{\prime}\right)(w(z):=)\left|M_{+}(z)+M_{-}(z)\right| \not \equiv 0$ on $\rho\left(A_{+, 0}\right) \cap \rho\left(A_{-, 0}\right)$.

$\left(A 3^{\prime}\right)$ There is $y_{1}>0$, such that for all $f_{A_{ \pm}} \in \mathcal{K}_{ \pm}$:

$$
\int_{0}^{y_{1}} \frac{\left|\widehat{f}_{A_{ \pm}}(\alpha+i y)\right|^{2}}{w(\alpha+i y)} d y<\infty, \quad \int_{0}^{y_{1}} \frac{\left|\widehat{f}_{A_{ \pm}}(\alpha-i y)\right|^{2}}{w(\alpha+i y)} d y<\infty .
$$

Then, the coupling $A_{1}$ of the operators $A_{+, 0}$ and $A_{-, 0}$ is a definitizable operator and

$$
\alpha \notin c_{s}(A) \text {. }
$$

Proof. In view of Corollary 3.6, the operator $A_{0}:=A_{+, 0}[\dot{+}] A_{-, 0}$ is definitizable. By Theorem 4.4, the assumption (A2') implies $\rho\left(A_{1}\right) \neq \emptyset$. Then by [41] the operator $A_{1}$ is also definitizable.

By the assumption $\left(\mathrm{A}^{\prime}\right) \alpha \notin c_{s}\left(A_{ \pm, 0}\right)$, then $\alpha \notin c_{s}\left(A_{0}\right)$. Since by Theorem 2.4 there is $y_{2} \in\left(0, y_{1}\right)$, such that:

$$
\int_{0}^{y_{2}}\left|\operatorname{Re}\left[\left(A_{0}-\alpha-i y\right)^{-1} f, f\right]_{\mathcal{K}}\right| d y<\infty \text { for all } f \in \mathcal{K},
$$

it remains to show that:

$$
\int_{0}^{y_{2}}|\mathcal{A}(f, \alpha+i y)| d y<\infty \text { for all } f \in \mathcal{K}
$$


where $\mathcal{A}$ is defined as in (4.19). The proof of this inequality is similar to that in Theorem 4.6 and is based on the assumption $\left(\mathrm{A}^{\prime}\right)$.

\section{Application to Sturm-Liouville operators with indefinite weights}

Consider the differential expression:

$$
\ell(f)(t):=\frac{\operatorname{sgn} t}{w(t)}\left(-\frac{d}{d t}\left(\frac{d f}{r(t) d t}\right)+q(t) f(t)\right) \text { for a.a. } \quad t \in \mathbb{R},
$$

where the coefficients $r, q$ and $w$ are real functions on $\mathbb{R}$ satisfying the conditions:

(C1) $r, q, w \in L_{\text {loc }}^{1}(\mathbb{R})$ and $r, w>0$ a.e. on $\mathbb{R}$,

(C2) the expression $\ell$ is in the limit point case at $-\infty$ and at $+\infty$.

Let $\mathcal{H}_{ \pm}=L_{w}^{2}\left(\mathbb{R}_{ \pm}\right)$be the standard weighted $L^{2}$-space with the positive definite inner product:

$$
(f, g)_{ \pm}=\int_{\mathbb{R}_{ \pm}} f(t) \overline{g(t)} w(t) d t \quad\left(f, g \in L_{w}^{2}\left(\mathbb{R}_{ \pm}\right)\right)
$$

Consider minimal differential operators $B_{ \pm}$generated by $\pm \ell$ in $L_{w_{ \pm}}^{2}\left(\mathbb{R}_{ \pm}\right)$, here $w_{ \pm}$denotes the restriction of $w$ to $\mathbb{R}_{ \pm}$. Since we assume that $\ell$ is in the limit point case at $\pm \infty$, the operator $B_{ \pm}$is a densely defined symmetric operator with defect numbers $(1,1)$ in the Hilbert space $L_{w_{ \pm}}^{2}\left(\mathbb{R}_{ \pm}\right)$and:

$$
\begin{aligned}
\operatorname{dom}\left(B_{ \pm}^{*}\right) & =\left\{f \in L_{w_{ \pm}}^{2}\left(\mathbb{R}_{ \pm}\right): f,\left(r^{-1} f^{\prime} \in A C_{\mathrm{loc}}[0, \pm \infty), \ell(f) \in L_{w_{ \pm}}^{2}\left(\mathbb{R}_{ \pm}\right)\right\}\right. \\
\operatorname{dom}\left(B_{ \pm}\right) & =\left\{f \in \operatorname{dom}\left(B_{ \pm}^{*}\right): f(0)=f^{\prime}(0)=0\right\} \\
B_{ \pm} f & := \pm \ell(f), \quad f \in \operatorname{dom}\left(B_{ \pm}\right) .
\end{aligned}
$$

In addition to $(\mathrm{C} 1),(\mathrm{C} 2)$, we assume that:

(C3) $B_{+}$and $B_{-}$are semibounded from below in $L_{w_{+}}^{2}\left(\mathbb{R}_{+}\right)$and $L_{w_{-}}^{2}\left(\mathbb{R}_{-}\right)$, respectively.

Let $z \in \mathbb{C} \backslash \mathbb{R}$ and denote by $\vartheta(\cdot, z)$ and $\varphi(\cdot, z)$ the unique solutions of the equation:

$$
-\left(r^{-1} f^{\prime}\right)^{\prime}+q f=z w f
$$

satisfying the boundary conditions:

$$
\varphi(0, z)=1,\left(r^{-1} \varphi^{\prime}\right)(0, z)=0 \quad \text { and } \vartheta(0, z)=0,\left(r^{-1} \vartheta^{\prime}\right)(0, z)=1 \text {, respectively. }
$$

Since we assume that $\pm \ell$ are in the limit point case at $\pm \infty$, for each $z \in \mathbb{C} \backslash \mathbb{R}$ there is a unique solution:

$$
\psi_{ \pm}(t, z)=\varphi(t, z) \pm m_{ \pm}(z) \vartheta(t, z), \quad t \in \mathbb{R}_{ \pm},
$$

of the restriction of $\pm \ell(f)=z f$ to $\mathbb{R}_{ \pm}$which belongs to $L_{w_{ \pm}}^{2}\left(\mathbb{R}_{ \pm}\right)$. Relation (5.3) defines the function $m_{ \pm}$: $\mathbb{C} \backslash \mathbb{R} \rightarrow \mathbb{C}$ uniquely. The function $m_{ \pm}$is called the Dirichlet $m$-coefficient of the restriction of the expression $\pm \ell$ to $\mathbb{R}_{ \pm}$.

A boundary triple for $B_{ \pm}^{*}$ is $\left\{\mathbb{C}, \Gamma_{0}^{ \pm}, \Gamma_{1}^{ \pm}\right\}$, where:

$$
\Gamma_{0}^{ \pm} f:=f(0 \pm), \quad \Gamma_{1}^{ \pm}(f)= \pm\left(r^{-1} f^{\prime}\right)(0 \pm), \quad f \in \operatorname{dom}\left(B_{ \pm}^{*}\right)
$$

It follows from (4.6) and (5.4) that the Dirichlet $m$-coefficient $m_{ \pm}$defined by (5.3) coincides with the Weyl function of the operator $B_{ \pm}$in (5.2) relative to the boundary triple in (5.4).

It is natural to consider the expression $\ell$ in the Krein space $\left(\mathcal{K},[\cdot, \cdot]_{\mathcal{K}}\right)$, where $\mathcal{K}=L_{w}^{2}(\mathbb{R})$ is the standard weighted $L^{2}$-space endowed with the indefinite inner product:

$$
[f, g]_{\mathcal{K}}=(J f, g)_{L_{w}^{2}(\mathbb{R})}=\int_{\mathbb{R}} \operatorname{sgn} t f(t) \overline{g(t)} d t, \quad f, g \in L_{w}^{2}(\mathbb{R}),
$$

and the operator:

is a fundamental symmetry on $\left(\mathcal{K},[\cdot, \cdot]_{\mathcal{K}}\right)$. We set:

$$
(J f)(t)=(\operatorname{sgn} t) f(t), \quad f \in L_{w}^{2}(\mathbb{R}),
$$

$$
\mathcal{K}_{ \pm}=\left\{f \in L_{w}^{2}(\mathbb{R}): f=0 \text { a.e. on } \mathbb{R}_{\mp}\right\} .
$$

Then $\mathcal{K}=\mathcal{K}_{+}[\dot{+}] \mathcal{K}_{-}$is the fundamental decomposition corresponding to $J$. 
Let the operators $A_{ \pm}:= \pm B_{ \pm}$be considered as semibounded symmetric operators in the Kreĭn spaces $\left(L_{w_{ \pm}}^{2}\left(\mathbb{R}_{ \pm}\right), \pm(\cdot, \cdot)_{L_{w_{ \pm}}^{2}\left(\mathbb{R}_{ \pm}\right)}\right)$. Then, the triples (5.4) are boundary triples for $A_{ \pm}^{+}$. The corresponding Weyl functions of the operators $A_{+}$and $A_{-}$take the form:

$$
M_{+}(z)=m_{+}(z), \quad M_{-}(z)=m_{-}(-z) .
$$

Consider a symmetric operator $A$ in the Kreĭn space $\left(\mathcal{K},[\cdot, \cdot]_{\mathcal{K}}\right)$ determined by the conditions (4.9). Then the domain of the adjoint operator $A^{+}$is characterized by the boundary condition (4.10), which in view of (5.4), takes the form:

$$
f(0+)=f(0-) .
$$

Consider the coupling $A_{1}$ of $A_{+}$and $A_{-}$relative to the boundary triples (5.4). $A_{1}$ is characterized by the boundary conditions (4.13), which now can be rewritten as:

$$
f(0+)=f(0-), \quad\left(r^{-1} f^{\prime}\right)(0+)=\left(r^{-1} f^{\prime}\right)(0-) .
$$

Therefore, the operator $A_{1}$ is associated with the expression in (5.1) in the Hilbert space $L_{w}^{2}(\mathbb{R})$; that is $A_{1} f=\ell(f)$ for all:

$$
f \in \operatorname{dom}\left(A_{1}\right)=\left\{f \in L_{w}^{2}(\mathbb{R}): f, r^{-1} f^{\prime} \in A C_{\mathrm{loc}}(\mathbb{R}), \ell(f) \in L_{w}^{2}(\mathbb{R})\right\} .
$$

Notice, that the assumption (A1) of Theorem 4.6 is satisfied in view of (C3) and the assumption (A2) is satisfied since if $m_{+}(z)+m_{-}(-z) \equiv 0$ then $m_{+}(z)=-m_{-}(-z)$ is holomorphic on the half-line $\left(-\beta_{-}, \infty\right)$, what is impossible for the $m$-coefficient of the Sturm-Liouville operator. These considerations and Theorem 4.6 justify the following:

Proposition 5.1. Let the differential operation $\ell$ satisfy (C1), (C2) and let the minimal differential operators $B_{ \pm}$ generated by $\pm \ell$ in $L_{w}^{2}\left(\mathbb{R}_{ \pm}\right)$satisfy (C3) and let $m_{ \pm}$be the Dirichlet $m$-functions of $B_{ \pm}$. Then, the coupling $A_{1}$ of $A_{+}$and $A_{-}$is locally definitizable in the Krein space $\left(\mathcal{K},[\cdot, \cdot]_{\mathcal{K}}\right)$. If, in addition, $m_{+}$and $m_{-}$satisfy the condition (4.18), then $\infty \notin c_{s}\left(A_{1}\right)$.

\section{Acknowledgements}

The research of the first author was supported by the Deutsche Forschungsgemeinschaft (DFG) under grant no. TR 903/16-1 and Ministry of Education and Science of Ukraine (projects \# 0115U000136, 0115U000556).

\section{References}

[1] Azizov T.Ya., Iokhvidov I.S. Linear operators in spaces with an indefinite metric, John Wiley \& Sons, 1990.

[2] Bognar J. Indefinite inner product spaces, Springer, 1974.

[3] Bender C.M., Brody D.C., Jones H.F. Complex extension of quantum mechanics. Phys. Rev. Lett., 2002, 89, P. 270401.

[4] Bender C.M., Brody D.C., Jones H.F. Must a Hamiltonian be Hermitian? Am. J. Phys., 2003, 71, P. $1095-1102$.

[5] Caliceti E., Graffi S., Sjöstrand J. Spectra of $\mathcal{P} \mathcal{T}$-symmetric operators and perturbation theory. J. Phys. A: Math. Gen., 2005, 38, P. 185-193.

[6] Günther U., Stefani F., Znojil M. MHD $\alpha^{2}$-dynamo, squire equation and $\mathcal{P} \mathcal{T}$-symmetric interpolation between square well and harmonic oscillator. J. Math. Phys., 2005, 46, P. 063504.

[7] Langer H., Tretter C. A Krein space approach to $\mathcal{P} \mathcal{T}$-symmetry. Czech. J. Phys., 2004, 54, P. $1113-1120$.

[8] Albeverio S., Günther U., Kuzhel S. J-self-adjoint operators with C-symmetries: extension theory approach. J. Phys. A: Math. Theor., 2009, 42, P. 105205.

[9] Caliceti E., Graffi S., Hitrik M., Sjöstrand J. Quadratic $\mathcal{P} \mathcal{T}$-symmetric operators with real spectrum and similarity to self-adjoint operators. J. Phys. A: Math. Theor, 2012, 45, P. 444007.

[10] Tanaka T. PT-symmetric quantum theory defined in a Krein space. J. Phys. A: Math. Gen., 2006, 39, P. L369-L376.

[11] Tanaka T. General aspects of PT-symmetric and P-self-adjoint quantum theory in a Krein space. J. Phys. A: Math. Gen., 2006, 39, P. $14175-14203$.

[12] Langer H. Verallgemeinerte Resolventen eines J-nichtnegativen Operators mit endlichem Defekt. J. Functional Analysis, 1971, 8, P. 287320.

[13] Jonas P. On a class of unitary operators in Krein space. Oper. Theory Adv. Appl., 1986, 17, P. 151-172.

[14] Jonas P. On a class of self-adjoint operators in Krein space and their compact perturbations. Integral Equations Operator Theory, 1988, 11, P. 351-384.

[15] Štraus A.V. Extensions and generalized resolvents of a symmetric operator which is not densely defined. Math. USSR-Izvestija, 1970, 4, P. 179-208.

[16] Calkin J.W. Abstract symmetric boundary conditions. Trans. Am. Math. Soc., 1939, 45, P. $369-442$.

[17] Kochubei A.N. On extentions of symmetric operators and symmetric binary relations. Matem. Zametki, 1975, 17, P. 41-48.

[18] Bruk V.M. On a class of problems with the spectral parameter in the boundary conditions. Mat. Sb., 1976, 100, P. $210-216$.

[19] Gorbachuk V.I., Gorbachuk M.L. Boundary value problems for operator differential equations, Kluwer Academic Publishers Group, 1991.

[20] Karabash I.M. J-self-adjoint ordinary differential operators similar to self-adjoint operators. Methods Funct. Anal. Topology, 2000, 6, P. 22-49. 
[21] Karabash I.M., Malamud M.M. Indefinite Sturm-Liouville operators with finite zone potentials. Operators and Matrices, 2007, 1, P. 301368.

[22] Karabash I.M., Kostenko A., Malamud M.M. The similarity problem for $J$-nonnegative Sturm-Liouville operators. J. Differential Equations, 2009, 246, P. 964-997.

[23] Kostenko A. The similarity problem for indefinite Sturm-Liouville operators and the HELP inequality. Adv. Math., 2013, 246, P. 368-413.

[24] Behrndt J. On the spectral theory of singular indefinite Sturm-Liouville operators. J. Math. Anal. Appl., 2007, 334, P. 1439-1449.

[25] Veselić K. On spectral properties of a class of J-self-adjoint operators I. Glasnik Mat. Ser., 1972, 7, P. $229-248$.

[26] Veselić K. On spectral properties of a class of J-self-adjoint operators II. Glasnik Mat. Ser., 1972, 7, P. $249-254$.

[27] Jonas P. Regularity criteria for critical points of definitizable operators. Operator Theory: Advances and Applications, 1984, 14, P. 179-195.

[28] Ćurgus B., Derkach V. Partially fundamentally reducible operators in Krè̆n spaces. Integral Equations Operator Theory, 2015, 82, P. 469-518.

[29] Ćurgus B., Langer H. A Kreĭn space approach to symmetric ordinary differential operators with an indefinite weight function. J. Differential Equations, 1989, 79, P. 31-61.

[30] Kac I.S., Krĕn M.G. R-functions-analytic functions mapping the upper halfplane into itself. Amer. Math. Soc. Transl. Ser., 1974, 103, P. $1-18$.

[31] Donoghue W.F. Monotone matrix functions and analytic continuation. Die Grundlehren der mathematischen Wissenschaften, Band 207, Springer-Verlag, 1974.

[32] Langer H. Spectral functions of definitizable operators in Kreĭn spaces. In Functional analysis: Lecture Notes in Math., Springer, 1982, 948, P. 1-46.

[33] Langer H. Spektraltheorie linearer Operatoren in J-Räumen und einige Anwendungen auf die Schar $L(\lambda)=\lambda^{2} I+\lambda B+C$. Habilitationsschrift, Technische Universität Dresden, 1965.

[34] Azizov T. Ya., Jonas P., Trunk C. Spectral points of type $\pi_{+}$and $\pi_{-}$of selfadjoint operators in Kreĭn spaces. J. Funct. Anal., 2005, 226, P. 114-137.

[35] Jonas P. On Locally Definite Operators in Kreĭn Spaces. In Spectral Theory and its Applications, Ion Colojoară Anniversary Volume, Theta, Bucharest, 2003, P. 95-127.

[36] Lancaster P., Markus A.S., Matsaev V.I. Definitizable operators and quasihyperbolic operator polynomials. J. Funct. Anal., 1995, 131, P. $1-28$.

[37] Langer H., Markus A.S., Matsaev V.I. Locally definite operators in indefinite inner product spaces. Math. Ann., 1997, 308, P. 405-424.

[38] Ando T. Linear Operators in Krein Spaces, Lecture Notes, Hokkaido University, Sapporo, 1979.

[39] Trunk C. Locally definitizable operators: The local structure of the spectrum. In: Operator Theory, D. Alpay (Ed.), Springer, 2015, P. 241-259.

[40] Behrndt J. Finite rank perturbations of locally definitizable self-adjoint operators in Krein spaces. J. Operator Theory, 2007, 58, P. 415-440.

[41] Jonas P., Langer H. Compact perturbations of definitizable operators. J. Operator Theory, 1979, 2, P. 63-77.

[42] Karabash I., Trunk C. Spectral properties of singular Sturm-Liouville operators. Proc. R. Soc. Edinb. Sect. A, 2009, 139, P. 483-503.

[43] Akhiezer N.I., Glazman I.M. Theory of Linear Operators in Hilbert Space, Dover Publications, 1993.

[44] Derkach V.A., Malamud M.M. Generalized resolvents and the boundary value problems for hermitian operators with gaps. J. Funct. Anal., 1991, 95, P. 1-95.

[45] Derkach V.A. On Weyl function and generalized resolvents of a Hermitian operator in a Krèn space. Integral Equations Operator Theory, 1995, 23, P. 387-415.

[46] Kreĭn M.G., Langer H. Über einige Fortsetzungsprobleme, die eng mit der Theorie hermitescher Operatoren in Raume $\Pi_{\kappa}$ zusammenhängen, Teil I: Einige Funktionenklassen und ihre Darstellungen. Math. Nachr., 1977, 77, P. 187-236.

[47] Derkach V.A., Hassi S., Malamud M.M., de Snoo H.S.V. Generalized resolvents of symmetric operators and admissibility. Methods Funct. Anal. Topol., 2000, 6, P. 24-55. 